\title{
Anabases
}

ANABASES Traditions et réceptions de l'Antiquité

$20 \mid 2014$

Varia

\section{Traduire les Géorgiques au rythme des saisons}

\section{Aymeric Münch}

\section{OpenEdition}

Journals

Édition électronique

URL : http://journals.openedition.org/anabases/5067

DOI : 10.4000/anabases.5067

ISSN : 2256-9421

\section{Éditeur}

E.R.A.S.M.E.

\section{Édition imprimée}

Date de publication : 1 novembre 2014

Pagination : 249-256

ISSN : 1774-4296

\section{Référence électronique}

Aymeric Münch, «Traduire les Géorgiques au rythme des saisons », Anabases [En ligne], 20 | 2014, mis en ligne le 01 novembre 2017, consulté le 20 octobre 2019. URL : http://journals.openedition.org/ anabases/5067 ; DOI : 10.4000/anabases.5067 


\section{Traduire les Géorgiques au rythme des saisons}

AyMeric MÜNCH

DANS nos sociéTÉs CitADINES, qui lirait encore les Géorgiques de Virgile, cet hymne au travail de la terre écrit par un des plus célèbres auteurs latins? Le jeune latiniste passera son chemin et lira avec plus d'appétit les aventures épiques d'Énée. Le cultivateur désireux de retrouver des techniques originelles, forcément plus respectueuses de la nature et des sols, considérera les références mythiques comme des ornements abscons. Il raillera la naïveté de l'auteur lorsqu'il s'émerveille, par exemple, de pommes poussant sur un poirier ou lorsqu'il évoque avec lyrisme les pouliches fécondées par le vent. L'amateur de mythologie sera frustré du caractère souvent elliptique des références... Faudrait-il être un érudit pour lire ce texte, dans l'isolement et le silence d'une bibliothèque ? Non, car la forme de ces vers est un appel à la voix, au souffle.

Virgile lui-même les a fait connaitre en 29 avant J.-C., lors d'une recitātiō, une lecture publique, certes devant un cercle restreint, aristocratique et lettré. Octave, de retour d'Actium ${ }^{1}$, en était probablement. À la noblesse du public répond la noblesse $\mathrm{du}$ vers, l'hexamètre dactylique, vers d'Homère, d'Hésiode et d'Ennius. Le projet, sans doute inspiré par le futur empereur, est de rendre aux Romains l'amour de leur terre, ravagée par les guerres civiles qui ont terni la République agonisante. Virgile ne souhaite pas rédiger un traité technique, Varron et Caton l'ancien s'en sont chargés. Il souhaite chanter en aède, comme Démodocos, comme Homère, et son sujet n'est autre que la terre de ses ancêtres, la terre nourricière d'Italie : 
...tibi rēs antīquae laudis et artis ${ }^{2}$

ingredior sānctōs ausus recludere fontīs,

Ascraeumque canō Rōmāna per oppida carmen ${ }^{3}$.

... j'aborde pour toi le sujet de ta gloire,

l'art ancestral, osant rouvrir les fontaines sacrales,

et je chante un chant ascréen dans les villes romaines.

L'épopée guerrière se devine derrière l'oppidum. Mais cette épopée sera surtout didactique. Virgile souhaite reprendre le flambeau d'Hésiode, poète d'Ascra et auteur des Travaux et les jours, qu'il traduit et adapte en maints lieux comme il traduit et adapte Homère ou le poète alexandrin Aratos. Il propose, avec le verbe ingredior, une démarche pas à pas, proprement pédagogique, vers une connaissance qu'il associe aux fontaines sacrées. Grandeur, gloire exaltée mais aussi mystère nimbent l'entreprise annoncée, quasi-initiatique.

Alors, comment rouvrir encore ces fontaines, deux mille ans après, comment créer des vers qui puissent être chantés par la parole et exalter encore cet amour de la terre et cette admiration pour celui qui la travaille ? Le paysan auquel s'adresse Virgile ne s'embarrasse pas de théories. Les connaissances qu'il possède de son ars antiqua sont empiriques et transmises de ses ancêtres. Le bon sens, le travail acharné et l'écoute des lois de la nature font le reste. Quant au traducteur, qui souhaite recreuser le sillon, le uersus virgilien, il obéira aux mêmes règles pour ce qui reste une pratique d'imprégnation et d'écoute, semblable au vieillard corycien qui

... transplanta en rangs espacés des ormes adultes.

Ille etiam sērās in uersum distulit ulmōś4.

Il doit tracer son sillon dans une terre au premier abord peu propice au rythme hexamétrique. Il écoute, encore et encore, les prédécesseurs de Virgile traduits en hexamètres français par ses propres prédécesseurs : l'Homère ${ }^{5}$ et l'Hésiode ${ }^{6}$ de Philippe Brunet, le Catulle d'André Markowicz ${ }^{7}$, le Lucrèce de Guillaume Boussard ${ }^{8}$. Puis il creuse ses six marques régulières où il placera des accents comme une rangée d'arbres. Ce processus de transplantation transforme les theseis, positions longues, impaires, de

2 Nous tenons à remercier Nicolas Lakshmanan, qui a marqué les voyelles longues dans les termes latins avec l'aide du logiciel Longiss, écrit par G. DE ROSNY, www.homeros.fr, 2011.

3 Virgile, Géorgiques, II, 174-176.

4 Virgile, Géorgiques, IV, 144.

5 Homère, L'Iliade, traduit du grec par Ph. BRUNET, Paris, 2010 ; L'Odyssée à paraître.

6 Hésiode, La Théogonie, Les Travaux et les Jours, fragments, traduction de Ph. BRUNET, commentaires de M-Ch. Leclerc, Paris, 1999.

7 Le livre de Catulle, traduit et présenté par A. MarKowiCZ, Lausanne, 1985.

8 À paraître. 
l'hexamètre originel frappées par l'ictus métrique en positions accentuées de diverses manières. En effet, la langue française offre pour cela des possibilités nombreuses car, d'une part presque tous les mots peuvent être ou non accentués sur leur dernière syllabe articulée et d'autre part certaines syllabes initiales ou internes peuvent bénéficier d'un accent. Cependant, de même que les syllabes longues en grec comme en latin ne possédaient sans doute jamais la même quantité, de même la phonologie du français dénombre plusieurs types et intensités d'accents.

Les possibilités de marquer les theseis du modèle métrique latin traduit en français se révèlent nombreuses. L'accent présenté comme le plus évident aussi bien par les phonéticiens, les métriciens que les spécialistes de la déclamation demeure lié à l'organisation syntaxico-sémantique de la phrase : il achève chaque unité de sens et peut parfois être souligné par une ponctuation. Mais, cette définition reste très large et peut s'appliquer à des éléments de tailles très diverses. Par exemple, le vers précédemment cité décrit une seule action et donne trois informations : quelle est cette action (transplanter), quel est son objet (des ormes adultes), comment elle est réalisée spatialement (en rangs espacés). Les deux derniers groupes syntaxiques sont composés d'un nom suivi d'un adjectif et précédé d'une préposition ou d'un article. Les adjectifs apportent des informations de nature à modifier le sens du verbe. On ne transplante pas de la même manière un jeune arbre et un arbre adulte, par exemple. Donc, un, trois ou cinq accents ? La dernière solution est séduisante mais elle ne contient pas les six accents attendus de l'hexamètre et surtout ne peut être rythmée avec des accents identiques entraînant des coupes de durée égale.

Le premier problème peut être résolu par deux théories phonétiques qui doteraient le verbe " transplanta » d'une attaque accentuelle sur la syllabe « trans- " : celle du " contre-accent ", censé frapper la première voyelle de chaque groupe syntaxico-sémantique, créant ainsi ce qu'I. Fonagy ${ }^{9}$ appelle un arc accentuel, ou celle du " contre-tonique ", développée par J. Mazaleyrat ${ }^{10}$ à l'image du nebenton allemand, qui frapperait la syllabe antépénultième des mots ou groupes de mots de trois syllabes ou davantage. Mais, en définitive c'est surtout la pratique de l'écriture et de la lecture hexamétrique qui finit par placer naturellement une attaque en début de vers. L'habitude du vers dactylique donne ainsi deux theseis à un mot lu naturellement avec deux atones suivies d'une tonique. Le ralentissement est facilité par la présence d'un son vocalique nasalisé suivi d'une sonante sur lequel la voix peut traîner. Est-ce là le retour d'une conception quantitative de la langue ? Non, mais simplement un réveil ponctuel de possibilités de diction immanentes. L'intonation naturelle se trouve ainsi légèrement transformée par

9 I. FonaGy, L'accent en français contemporain, Ottawa, 1979, p. 142. Plus récemment, $\mathrm{Ph}$. MARTIN précise que cet accent secondaire frappe la première syllabe de l'unité de classe ouverte (verbe, nom, adjectif ou adverbe) du groupe (Intonation du français, Paris, 2009, p. 110).

10 J. Mazaleyrat, Éléments de métrique française, Paris, 1974. 
l'artifice de la diction. La règle de conservation du e féminin entre deux consonnes dans l'alexandrin classique n'est pas moins contraignante.

Le second problème trouve sa solution dans la hiérarchie accentuelle. "Rangs " et " ormes " peuvent être accentués sans nécessairement occasionner une coupe. Ainsi, sur les six accents les accents pairs sont des accents de groupes syntaxiques et les accents impairs sont des marques soit purement phonétiques (le premier) soit des accents de mots réveillés mais de moindre intensité ou longueur que les accents syntaxiques.

Reculons de quelques pas pour observer la rangée d'arbres et voir l'alternance d'accents majeurs et d'accents mineurs, les accents dit majeurs engendrant les coupes trihémimères et hephthéminères :

transplantạ // en rangs espacés // des ormes adultes.

Ainsi, peu à peu, de même que la terre donne vie à la plante et la plante enrichit la terre, l'agencement des mots crée le rythme du vers et la forme métrique réveille le rythme des mots...

Mais, une fois le sillon préparé, les arbres plantés, il reste tout un travail de taille de la plante pour qu'elle retrouve sous ce nouveau climat la même vigueur que sous son climat natal. L'hexamètre ne suffit pas à rendre Virgile.
Ō fortūnātōs nimium, sua sì bona nō rint,
SS DD DS ${ }^{11}$
agricolās! quibus ipsa procul discordibus armīs
DD DS DS
fundit humō facilem ū̄ctum iustissima tellūs ${ }^{12}$.
DD SS DS
Mille fois bienheureux s'ils connaissaient leurs richesses,
SD SS DS
Ô campagnards ! quand pour eux, de la terre mêeme, le jüste
DD DS DS
Sol , loin des a armes rebellles, répand des vivires faciles !
DD DS DS

Avant toute traduction du premier vers, devenu proverbial, le travail commence par une lecture attentive de tous les effets sonores du texte original ; il peut s'intéresser à la distribution des consonnes et des voyelles, au jeu des résolutions des positions ancipitēs (qui peuvent être occupées par une syllabe longue ou une syllabe brève) et à la place des accents de mots par rapport aux theseis. Le début du vers est marqué par l'exclamation " $\bar{O}$ ", par la lenteur spondaïque, par la mise en valeur de l'adjectif «fortūnātōs » avant la coupe penthémimère ; le premier vers vibre doucement au gré d'une savante allitération de nasales $(\mathrm{n} / \mathrm{n} / \mathrm{m} / \mathrm{m} / \mathrm{n} / \mathrm{n} / \mathrm{in}$ ) et grâce à la faible présence d'occlusives sourdes (les deux $t$ de "fortūnātōs ", la finale de "nōrint ", perdue dans le silence suivant le vers); ce même vers joue avec les voyelles accentuées naturellement, obligeant la voix à partir d'un $o$ fermé, s'ouvrir sur un $a$ long et bien ouvert, puis un $i$ bref, nécessitant un resserrement de la gorge, qui s'affirme en $i$ long après le $u$, pour finir par le retour du $o$, d'abord bref,

11 Nous donnons la scansion de ces vers, avec $\mathrm{D}$ pour dactyle et $\mathrm{S}$ pour spondée.

12 Virgile, Géorgiques, II, 458-460. 
puis long; enfin le rejet du substantif "agricolās " au début du vers suivant et mis en valeur par la coupe trihémimère qui le suit. Le musicien observerait donc un vers marqué par la douceur de son articulation, un effet de crescendo / decrescendo mettant en valeur un "fortūnātōs » feutré et un «sī " plus appuyé, sommet intensif du vers, et en direction duquel les deux brèves de l'adjectif possessif « sua " placées après la coupe hephthémimère, se précipitent. Il est donc une condition essentielle au bonheur de ces cultivateurs : «bona nōrint ", qu'ils se rendent compte de leurs biens.

Après la lecture viennent les nombreux tâtonnements autour du schéma à six accents, six marques ou six proéminences, tous espacés par un ou deux temps. Ce schéma s'achève, pour rendre au spondée final son rythme descendant, par une finale féminine. Une première version, correcte rythmiquement, donnerait : « Ô, trop fortunés, s'ils connaissaient leurs richesses, / agriculteurs ". La syntaxe est respectée ainsi que l'ordre des mots, dans la mesure du possible. "sua bona" se trouve rendu non par " leurs biens " mais « leurs richesses", au sens large, pour respecter la finale spondaïque. Cependant, la disjonction " fortunés [...] agriculteurs! " choque la syntaxe française, sans parler du jeu des voyelles (deux $o$ fermés suivis d'un $o$ ouvert, pour commencer), qui devient grossier ni de l'alliance "fortunés " " richesse " qui laisse à croire que ces agriculteurs ont gagné à la loterie... La présence des spondées est trop importante, donnant de la lourdeur au vers, tandis que le latin offrait une alternance (deux spondées suivis de deux dactyles avant la clausule). Il faut alors chercher de nouvelles solutions quitte à s'écarter un peu du modèle.

La proposition définitive, "Mille fois bienheureux s'ils connaissaient leurs richesses, / Ô campagnards !... " offre les avantages suivants : la disjonction disparait au profit d'une exclamation en incise, marquée par le " $O$ " qui se décale d'un vers mais reste en première place. Le " $\bar{o}$ " latin et le " nimium " fusionnent dans l'hyperbole exclamative " mille fois ». Une seule occlusive vient heurter l'oreille. Le " $s \bar{\imath}$ » occupe une position d'arsis et non de thêsis, ce qui le met moins en valeur, mais il reste seul sur sa position et libre au récitant d'allonger artificiellement les arseis monosyllabiques... La traduction d' "agricolās" pose problème, car, pour garder le « $\bar{O}$ ", il ne reste plus que trois temps à occuper. "Laboureur " est trop spécifique, "campagnard " trop général, mais il a le mérite de contenir le sens d'ager, le champ.

Tout serait affaire de négociation, dans cet univers où seules six idées au maximum par vers peuvent être clairement distinguées, étant entendu qu'en français chaque accent achève une idée. Serait-ce alors le règne des mots courts et denses sémantiquement ? Par exemple, notre Virgile, avec une abondance de monosyllabes, "chante un chant ascréen dans les villes romaines " et non les "citadelles romaines ", plus épiques, mais il pose sur deux accents différents le polyptote " chante "/ chant " qui répond à celui du latin, " cano»/« carmen »... À l'inverse, le mot de trois syllabes, ou davantage, s'il occupe parfois deux accents, peut être choisi pour exprimer, outre son contenu sémantique et selon le contexte, l'idée de grandeur, de langueur, ou de souplesse... Nous avons souligné combien l'effet de mîmêsis rythmique commence dès le premier mot, trisyllabique, du vers consacré à l'action de l'horticulteur laborieux. 
Revenons aux agriculteurs :

quibus ipsa procul discordibus armīs

fundit humō facilem uīctum iustissima tellūs.

Cette fin de phrase comporte une forte disjonction, ipsa... iustissima tellūs (« luimême... le très juste sol ") et une mise en valeur du sujet de la proposition relative dans la dernière clausule. Le traducteur ne se pose plus alors dans l'alternative du mot à mot ou de l'élégance française mais se demande comment répondre rythmiquement à un problème somme toute rythmique : une disjonction ou attente d'une résolution syntaxique. Le vers français sait aussi créer des attentes par le principe du rejet, qui implique une suspension de la voix plus ou moins longue selon l'orateur. Ici, l'adjectif " juste " peut résonner en fin de vers, marquage sonore qui remplace le marquage sémantique du superlatif latin. L'attente du substantif est résolue quand la suspension retombe sur le "sol ", monosyllabe dont l'accent est renforcé par sa position en thesis initiale. La traduction de l'adjectif ipsa, placé en début de proposition pour en annoncer la fin peut sembler alors superflue. Nous avons choisi de le conserver mais de l'associer à la " terre ", créant ainsi une homophonie entre " terre " et " même ".

Mais alors, comment transposer l'opposition marquée par Virgile entre un vers consacré à la guerre et le suivant consacré à la terre, le premier marqué par des occlusives sourdes et le roulement de l'allitération en /r/ (quibus ipsa procul discordibus armīs) et le suivant par les nasales, et la douce anaphore du /f/ (fundit humō facilem uīctum iustissima tellüs) ? Là encore, la solution est rythmique. Observons les passages consacrés à la terre : " de la terre même le juste/ sol " "répand des vivres faciles ". En dehors $\mathrm{du}$ cadre hexamétrique et en conservant l'articulation classique du /e/ féminin entre deux consonnes, le rythme obtenu est le suivant : $u u-u-u u-u /$-, puis $u-u-u u-u^{13}$. Les monosyllabes de forme féminine (" terre ", " même ", " juste ", " vivre ») bénéficient d'un allongement de la tonique pour compenser la brièveté du /e/ muet, comme l'ont remarqué J.-C. Milner et F. Regnault ${ }^{14}$, dans un ouvrage consacré à la déclamation de l'alexandrin, et, bien avant, le poète humaniste J. de la Taille ${ }^{15}$ dans une des premières théories du vers "mesuré " à la française. D'autre part, l'alternance thesis / arsis de l'hexamètre imposant en français une syllabe marquée en thesis et une ou deux non marquées en arsis, n'interdit pas la possibilité de remplir une arsis par un /e/ muet, c'est-à-dire, de remplir une position résolue comme longue par une voyelle atone et particulièrement brève. Dans ce cas, l'allongement compensatoire de la tonique est d'autant plus sensible, si l'interprète choisit de ne pas appuyer outre mesure un son

13 Nous marquons les toniques par des - et les atones par des $u$.

14 J.-C. Milner, F. Regnault, Dire le vers, court traité à l'intention des acteurs et des amateurs d'alexandrins, Paris, 2008 (1987), p. 59.

15 J. de LA TAILlE, La manière de faire des vers en François, comme en Grec et en Latin, Paris, 1573, p. 7. 
naturellement élidé. L'effet est sensiblement identique lorsque un clitique, comme l'article indéfini « des ", remplit à lui seul une arsis: « répand des vivres faciles». La tonique en thesis qui précède peut pour la même raison se trouver allongée. En revanche, aucun appui de la sorte ne s'observe dans le passage consacré à la guerre : "loin des armes rebelles ", rythmé $u u-u u-u$, ou les /e/ féminins et le clitique occupent une position brève en arsis du dactyle. Ainsi, tout en restant très proche de la distribution dactyle / spondée du texte originel, la traduction quitte le mot à mot pour retrouver une autre forme de marquage rythmique des unités sémantiques.

Maintenant, les sillons sont tracés, l'attente de la tonique après une ou deux atones est instituée dans l'oreille du traducteur et le texte tourne autour du schéma rythmique comme les vignes autour des ormes, créant retards et accélérations, tensions dues autant au naturel de la langue qu'aux choix déclamatifs. La joie de la profération aédique peut alors embrasser aussi bien une liste de fruits, olives et poires, que le catalogue des nymphes entourant Cyrène.

Les olives grasses naissent sous plus d'un visage :

Les orchades, les allongées, les pressées, baies amères,

Comme les fruits des forêts phéaciennes. L'on voit des boutures

De poires crustumériennes, syriennes et lourdes palmaires ${ }^{16}$.

Forestière, Dorée, Mélodieuse, Amie-des-feuillages,

Dont les cheveux lumineux recouvraient leur nuque splendide,

Grâce-des-îles, Creusée, Florissante, Amie-de-la-vague,

Fière-cavale et la blonde Louve-des-bois - l'une vierge,

L'autre venant de connaitre le travail de Lucine,

Gloire et sa sœur Abondance, les deux, des Océanides,

Les deux, couvertes de peaux tachetées, les deux, de dorures,

Et Corinthe, Hyperborée, Chasseresse l'Asiate

Et la véloce Source-d'Arès, enfin loin de ses flèches ${ }^{17}$.

Agronomie et mythologie se réconcilient enfin dans cette suite de noms parfois traduits et imagés, comme les "lourdes palmaires ", qui remplissent la paume de la main, ou "Grâce-des-îles " qui pour l'auditeur francophone évoque un exotisme langoureux, parfois saisis dans leur étrangeté, comme les orchades ou Hyperborée... Tous les noms, souvent longs et sonores, doivent entrer dans le moule métrique, alors, qu'importent s'ils se bousculent, s'ils n'entrent pas dans le même ordre que leur version latine pourvu que ce soit avec la solennité que leur impose le rythme épique et qu'ils créent entre eux des résonances, de subtils échos : le son vocalique qui achève "crustumériennes ", "syriennes " et " palmaires ", dans un vers dominé par une allitération en

16 Virgile, Géorgiques, II, 85-88.

17 Virgile, Géorgiques, IV, 436-444. 
$/ \mathrm{r} /$; le retour du son $/ \mathrm{a} /$ et le frottement des doubles consonnes en début de mot dans "Grâce-des-îles, Creusée, Florissante, Amie-de-la-vague ".

Pour répondre enfin à notre première question, celle de savoir qui lirait aujourd'hui les Géorgiques, nous répondrons que si leur lecture purement intellectuelle, à la recherche d'un sens et d'une structure, à la recherche de conseils efficaces, à la recherche d'une histoire des techniques agricoles, etc., reste une épreuve, leur écoute, en latin comme en français mesuré, permet de saisir avec force et jubilation le dynamisme du propos virgilien, qui donne à voir, une suite d'images animées, là le labour, là la sieste sous l'arbre, là les abeilles bourdonnant, au rythme et à la lumière du temps et des saisons.

Aymeric MüNCH

ERIAC (Université de Rouen)

Faculté des Lettres et Sciences humaines

76821 Mont-Saint-Aignan cedex

aymeric.munch@ac-creteil.fr

\section{Quelques indications bibliographiques}

1519 Virgile, Géorgiques, G. Michel dit de Tours, Paris [première traduction française, en décasyllabes].

1552 Virgile, Enéide, chants 4 et 6, J. Du Bellay, Paris [en décasyllabes].

1555 Virgile, Géorgiques, R. Le Blanc, Paris [en décasyllabes].

1770 Virgile, Géorgiques, J. Delille, Paris [en alexandrins].

1944 Virgile, Bucoliques, P. VAléry, Paris [en alexandrins].

1948 Virgile, Géorgiques, P. A. Nicolas, Paris [hétérométrie].

1964 Virgile, Enéide, P. KLossowski, Paris [en vers libres].

2007 Virgile, Bucoliques, Géorgiques, J.-P. Chausserie-Laprée, Paris [en alexandrins].

Présentations scéniques de la traduction des Géorgiques en hexamètres, par Aymeric Münch :

2000 Orphée, spectacle de la compagnie Démodocos, mise en scène de Ph. Brunet, Tours, Paris et Vaison-la-Romaine.

2011 Rhapsodie printanière, récital voix (Aymeric Münch), violoncelle (Jonas Bouaniche) et piano (Valentin Cotton), festival des Dionysies, Réfectoire des Cordeliers.

2012Aristée, fantaisie mythologique d'après le livre quatre des Géorgiques, récital d'Aymeric Münch accompagné d'élèves du Lycée Eugène Delacroix de Drancy, festival des Dionysies, Réfectoire des Cordeliers. 